\title{
Efficient noise averaging in color holography by integration on shifted Fourier spectra
}

\author{
I. Ducin, K. Kakarenko, M. Makowski, ${ }^{*}$ A. Kowalczyk, M. Bieda and J. Suszek \\ Faculty of Physics, Warsaw University of Technology, Koszykowa 75, 00-662 Warszawa
}

Received August 13, 2014; accepted September 02, 2014; published September 30, 2014

\begin{abstract}
Modern holography is dealing mainly with speckle noise and computational complexity. A compromise is required between computational complexity (computing time) and quality of obtained images. This leads to a need to obtain a simple method of noise suppression with low computation complexity. An improved efficient projection of color images is presented. It uses time averaging of intensity patterns reconstructed from the same Fourier hologram with lateral shifting in $\mathrm{x}$ and $\mathrm{y}$ direction. We try to establish the optimal step of shifting the Fourier spectra in both directions for each of the primary colors.
\end{abstract}

The two main problems in holographic projection are high speckle noise and computational complexity. Numerous methods have been proposed to simplify the calculation and to suppress speckle noise [1-2]. Nevertheless, most of the previously proposed methods cannot overcome both of the mentioned problems at the same time.

The purpose of this work is to describe and evaluate numerically and experimentally a simple method of noise suppression with low computation requirements. The method is based on the previously reported shifted Fourier spectra [3], but it assumes detailed shifting optimization of the Fourier spectra.

Recently Golan and Shoham proposed a simple and efficient method of speckle suppression by time averaging of intensity patterns reconstructed from the same Fourier hologram with lateral shifting in the $x$ and $y$ direction [3].

That method utilizes the fact that intensity patterns reconstructed from Fourier holograms are insensitive to lateral position of the hologram with respect to the lens. On the other hand, phase relations change with lateral shifts leading to different speckle distributions on the projection screen. By time-integration of numerous such distributions significant noise reduction was obtained [3].

In this contribution, we take advantage of this method, but we try to establish the optimal step of shifting the spectra in both directions for red, green and blue illumination. We performed a numerical simulation of such a noise averaging process on matrices of $2048 \times 2048$ points. We iterated a Fourier hologram with 10 iterations in the Gerchberg - Saxton algorithm [4].

\footnotetext{
*E-mail: mcovsky@if.pw.edu.pl
}

The hologram contained the standard USAF - 1951 test pattern. After that we recorded the intensity pattern reconstructed form the hologram. Additionally, we recorded such distribution from the same hologram but shifted laterally in $\mathrm{x}$ and $\mathrm{y}$ directions by the some distance measured in pixels.

This distance will be referred to as a "step". Intensity distributions were added together to form a final noiseaveraged image. This image was then analyzed in terms of noise ratio. The noise ratio was defined as a standard deviation of intensity in a test bright region divided by the average value of intensity in the same region. Figs. 1-3 show the results of the described numerical simulations for 9,16 and 25 integrated holograms.

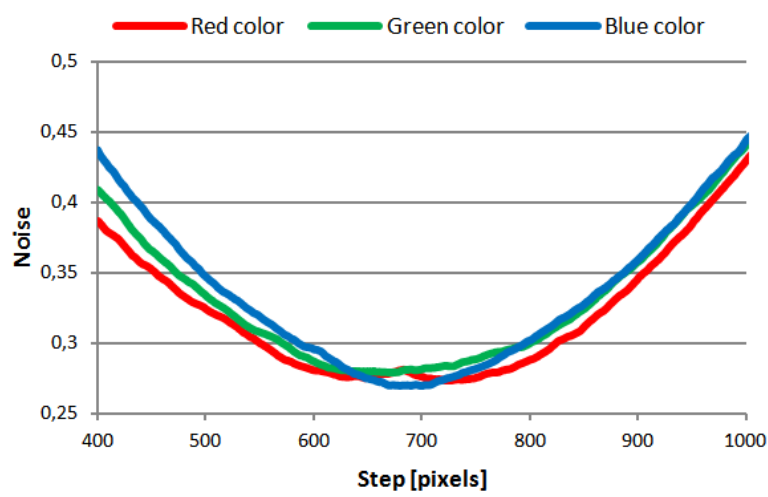

Fig. 1. Numerical simulations for 9 integrated shifted Fourier holograms for red, green and blue illumination.

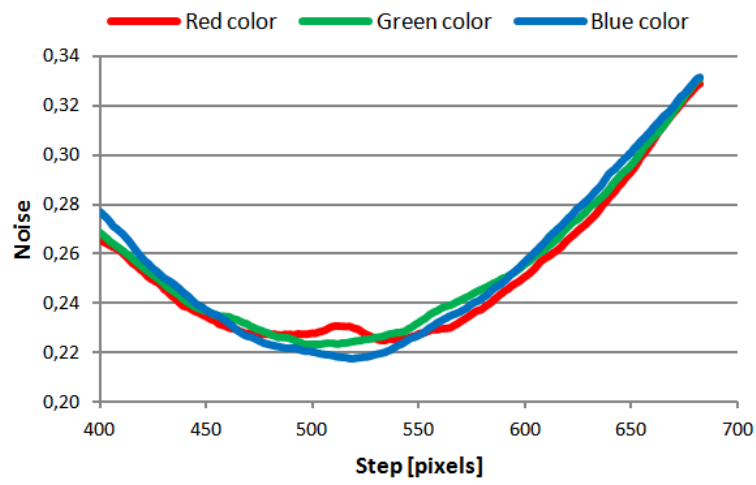

Fig. 2. Numerical simulations for 16 integrated shifted Fourier holograms for red, green and blue illumination. 


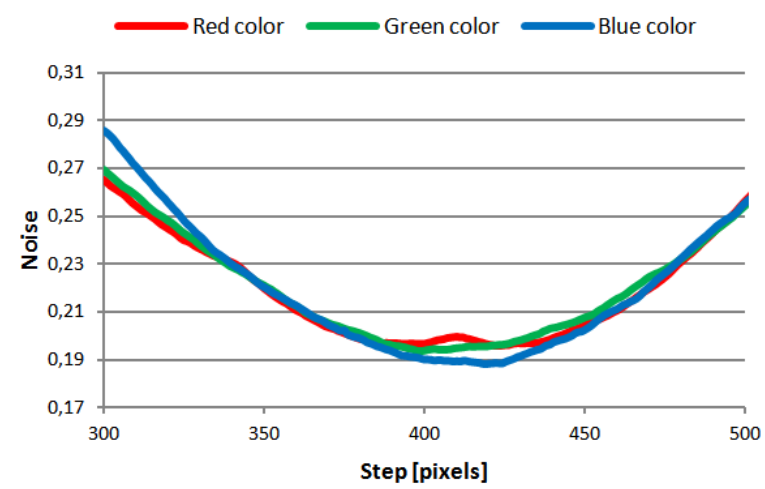

Fig. 3. Numerical simulations for 25 integrated shifted Fourier holograms for red, green and blue illumination.

The minimal value of noise occurs for a certain step value which is different for each primary color used. The optimal values are gathered in Tab. 1. Based on this conclusion, we compared the averaged final images obtained with the optimal step, with a fixed non optimized step value of 10 pixels and with the classic TDRP (Time Depended Random Phase) method [5].

Tab. 1 Optimal value of step for red, green and blue color for 9, 16, 25 integrated Fourier hologram.

\begin{tabular}{|c|c|c|c|}
\hline \multirow{2}{*}{$\begin{array}{c}\text { Number of } \\
\text { integrated holograms }\end{array}$} & \multicolumn{3}{|c|}{ Step [px] } \\
\cline { 2 - 4 } & Red & Green & Blue \\
\hline 9 & 721 & 676 & 700 \\
\hline 16 & 533 & 498 & 518 \\
\hline 25 & 422 & 399 & 419 \\
\hline
\end{tabular}

The sequence of lateral shift of the Fourier spectrum for the proposed method is presented in Fig. 4. The figure shows the case of the 9 integrated holograms for clarity, nevertheless, the same scheme was used for the cases of 16 and 25 integrated holograms. In a classic TDRP method the shifting sequence was simple, as presented in Fig. 5.

The results are shown in Figs. 6-8 for wavelength of $671,532,445 \mathrm{~nm}$, respectively.

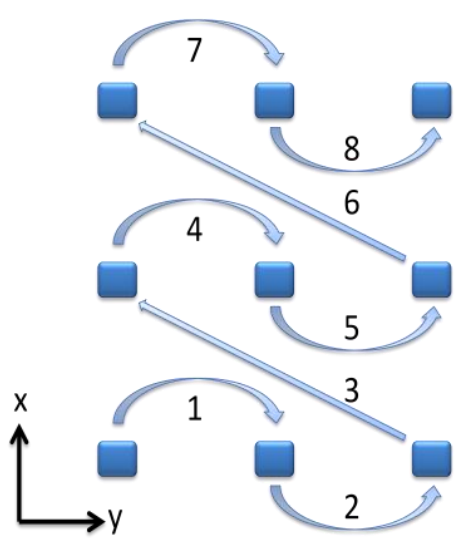

Fig. 4. The sequence of lateral shift for the proposed method.

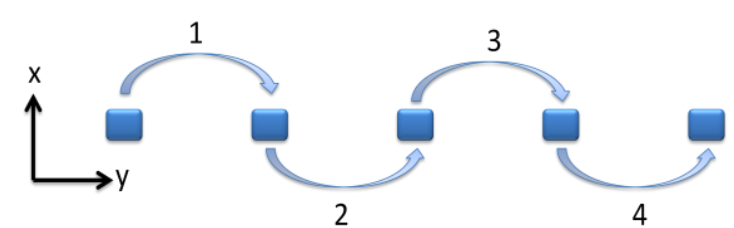

Fig. 5. The sequence of lateral shift in a classic TDRP method.

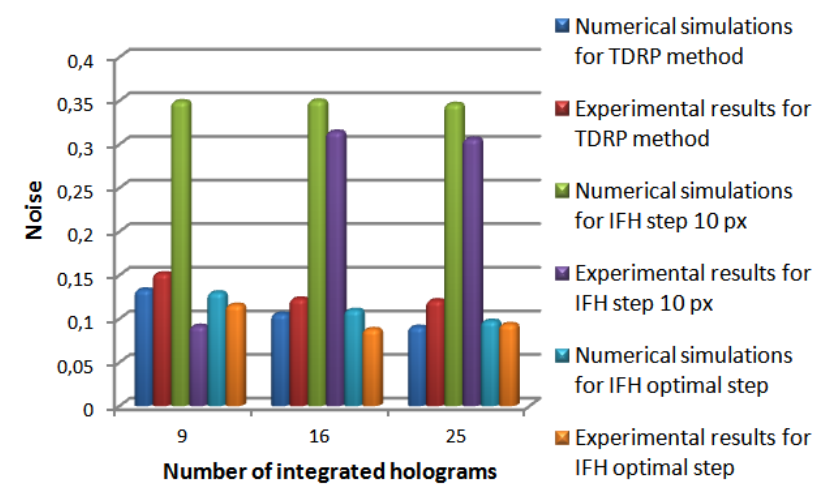

Fig. 6. Results for the wavelength of $671 \mathrm{~nm}$ for a classic TDRP method, integrated Fourier holograms shifted with a non - optimized step (IFH step $10 \mathrm{px}$ ) and integrated Fourier holograms shifted with an optimized step (IFH optimal step).

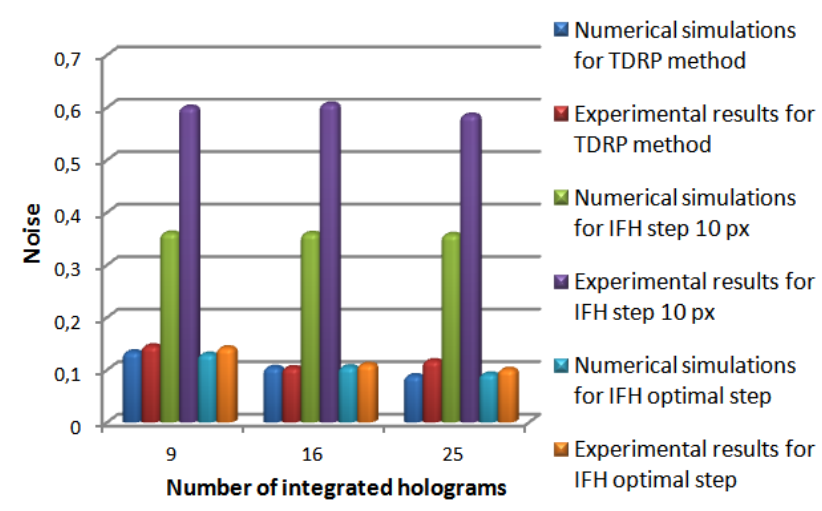

Fig. 7. Results for a wavelength of $532 \mathrm{~nm}$ for a classic TDRP method, integrated Fourier holograms shifted with a non - optimized step (IFH step $10 \mathrm{px}$ ) and integrated Fourier holograms shifted with an optimized step (IFH optimal step).

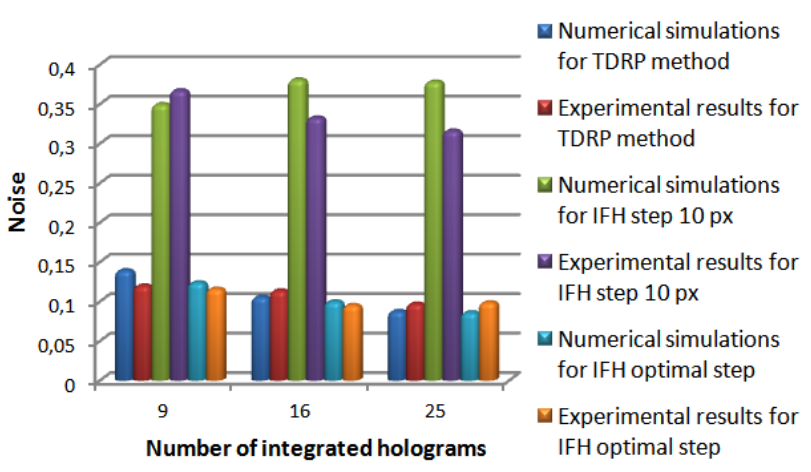

Fig. 8. Results for a wavelength of $445 \mathrm{~nm}$ for a classic TDRP method, integrated Fourier holograms shifted with a non - optimized step (IFH step 10 px) and integrated Fourier holograms shifted with an optimized step (IFH optimal step). 
Additionally, we present a composition of primary colored images into a white final image in Fig. 9. It can be seen that the proposed method has similar efficiency regarding noise suppression compared to a classic TDRP method.

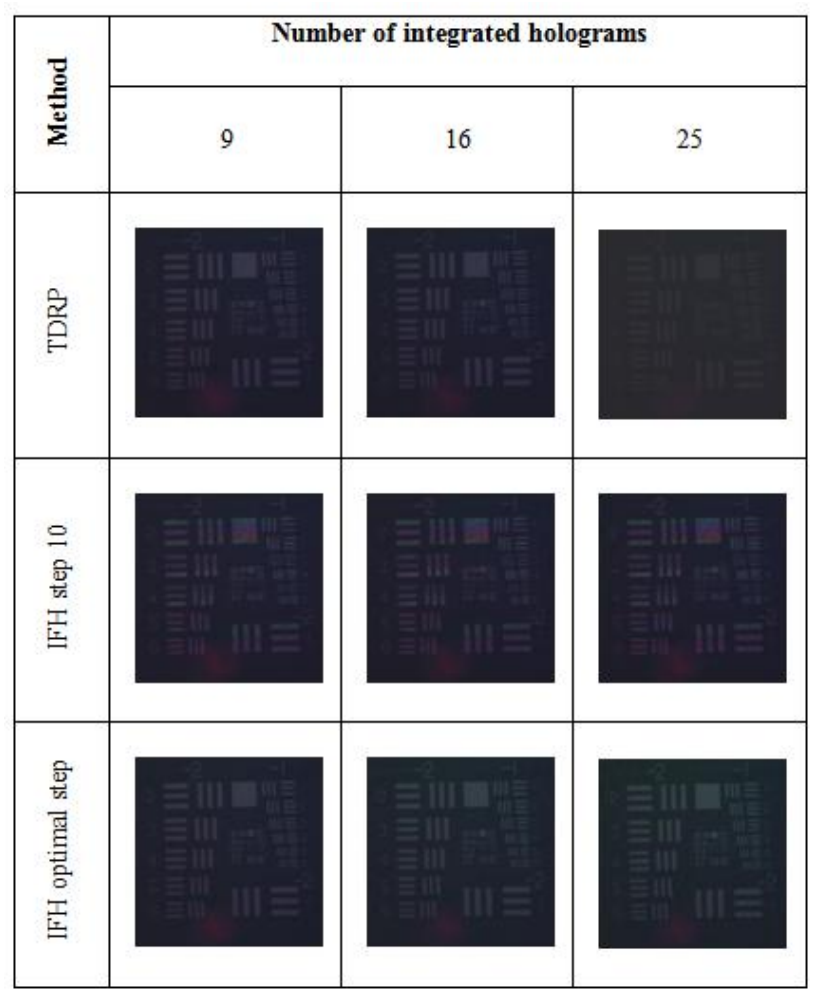

Fig. 9. Experimental results.

The problem lies in the fact that in the TDRP method one needs to calculated and iterate separately 9,16 or 25 different holograms of the same object. On the other hand, in our method one needs only to calculate and iterate one Fourier hologram, which gives us a significant time advantage. Additionally, the graphs in Fig. 6, Fig. 7 and Fig. 8 show that finding an optimized step value is very important to minimize the noise ratio.

Modern GPU processing of computer-generated holograms (CGH) [6-7] allow real time holographic projection. Nevertheless, for efficient noise averaging in a classic way it is necessary to calculate approximately 25 times more holograms as compared to the number of frames of the displayed animation. This requirement is too strong for medium class GPU processors, especially for handheld devices with strict consumption limits of electric power.

The utilization of a single hologram per animation frame proposed in this work allows a significant reduction in power requirements of the GPU processor without sacrificing image quality. The lateral shifting of the calculated hologram shows some similarities to the optical method of speckle reduction by fast rotating a matt plate in the optical setup.

The method is also suitable for future holographic projection devices with image resolution beyond Full HD.

This work was supported by the Polish National Center for Research and Development grant LIDER/013/469/L4/12/NCBR/2013.

\section{References}

[1] M. Makowski, Opt. Expr. 21, 29205 (2013).

[2] M. Makowski, A. Siemion, I. Ducin, K. Kakarenko, M. Sypek, A.M. Siemion, J. Suszek, D. Wojnowski, Z. Jaroszewicz, A. Kolodziejczyk, Chinese Opt. Lett. 9(12), 120008 (2011).

[3] L. Golan, S. Shoham, Opt. Expr. 17, 1330 (2009).

[4] R.W. Gerchberg, W.O. Saxton, Optik 35, 237 (1972).

[5] A. Czerwiński, K. Kakarenko, M. Sypek, M. Makowski, I. Ducin, J. Suszek, A. Kolodziejczyk, J. Bomba, Opt. Lett. 37(22), 4723 (2012).

[6] T. Shimobaba, T. Ito, N. Masuda, Y. Ichihashi, N. Takada, Opt. Expr. 18, 9955 (2010).

[7] T. Shimobaba, Y. Sato, J. Miura, M. Takenouchi, T. Ito, Opt. Expr. 16, 11776 (2008). 